\title{
Avaliação do tocoferol no congelamento do sêmen bovino e nas taxas de prenhez após inseminação artificial em tempo fixo*
}

\section{Tocopherol evaluations at the freezing of bovine semen on the pregnancy rates after fixed time artificial insemination}

Moacir Ferreira Duarte Júnior, ${ }^{* *}$ Luciana Keiko Hatamoto Zervoudakis, ${ }^{* * *}$ Joanis Tilemahos Zervoudakis, ${ }^{* * * *}$ Marcílio Nichi, ${ }^{* * * *}$ Ricardo Pimenta Bertolla, ${ }^{* * * * *}$ Pedro Paulo Tsuneda, ${ }^{* * * * * *}$ Luís Eduardo Senra e Silva, ${ }^{* * * * * *}$ Fabiana Mariani Wingert, ${ }^{* * * * * *}$ Walter Augusto dos Santos Marinho********

\begin{abstract}
Resumo
O presente estudo avaliou se a adição de tocoferol no diluidor para a criopreservação de sêmen bovino reduz os danos causados pelo estresse oxidativo e melhora a capacidade fertilizante do sêmen utilizado na IATF. No primeiro lote sincronizado foram utilizadas 84 fêmeas Nelore (Bostaurusindicus) e no segundo lote 44 fêmeas. Para a inseminação artificial as vacas foram divididas aleatoriamente em dois grupos: controle (inseminadas com sêmen criopreservado sem uso de aditivos) e tocoferol (inseminadas com sêmen criopreservado com adição de $10 \mathrm{mmol} / \mathrm{mLtocoferol}$ ). Foram utilizados sêmen de três reprodutores da raça Nelore (Bos taurus indicus), sendo as doses provenientes de uma única partida, distribuída aleatoriamente entre as fêmeas inseminadas. O diagnóstico de gestação foi realizado 35 dias após a IATF por exame ultrassonográfico retal. O experimento foi realizado em delineamento inteiramente casualizado, e a taxa de prenhez comparada pelo teste qui-quadrado. $\mathrm{A}$ adição de $10 \mathrm{mmol} / \mathrm{mL}$ de tocoferol no diluidor do sêmen não influenciou $(P>0,05)$ na taxa de prenhez após IATF em comparação ao grupo controle (sem aditivos) para as médias do primeiro lote sincronizado ( $n=84 ; 38,5 \%$ vs $40 \%$ ), segundo lote sincronizado ( $n=44 ; 28 \%$ vs $31,6 \%$ ) e para a média geral dos lotes ( $n=128 ; 34,4 \%$ vs $37,5 \%$ ). Nas condições experimentais a adição de $10 \mathrm{mmol}$ de tocoferol ao meio crioprotetor do sêmen não melhorou a taxa de prenhez após a inseminação artificial em tempo fixo na espécie bovina.
\end{abstract}

Palavras-chave: vitamina $\mathrm{E}$, espermatozoide, espécies reativas ao oxigênio, estresse oxidativo.

\begin{abstract}
The present study evaluated if the addition of tocopherol to the extender semen cryopreservation reduces the damage caused by oxidative stress and preserves the fertilizing capacity of semen used in FTAI. In the first lot we used 84 synchronized females Nelore (Bos taurus indicus) and the second lot 44 females.For the artificial insemination the cows were randomly divided into two groups: control (inseminated with semen cryopreserved without using additives) and treatment (inseminated with semen cryopreserved with added $10 \mathrm{mmol} / \mathrm{ml}$ tocoferol). We used three semen sires Nelore (Bos taurus indicus), with doses starting from a single, randomly distributed among females inseminated. The pregnancy diagnosis was done 35 days after FTAI by rectal ultrasonography. The experimental design was completely randomized and pregnancy rates compared by chi-square test. There were no effect $(P>$ 0.05 ) in pregnancy rate using cryopreserved semen with added $10 \mathrm{mmol} / \mathrm{ml}$ tocopherol in the bovine semen extender compared to the control group (no additives) in the first synchronized group ( $n=84,38.5 \%$ vs $40 \%$ ), in the second synchronized group ( $n=44$, $28 \%$ vs $31.6 \%$ ) and the all animals ( $n=128,34.4 \%$ vs $37.5 \%)$. We conclude that, under the experimentalconditions, the addition of $10 \mathrm{mmol}$ of tocopherol in the semen extender did not improve the pregnancy rate after FTAI in the bovine species.
\end{abstract}

Keywords: vitamin E, spermatozoon, reactive oxygen species, oxidative stress.

\section{Introdução}

A boa qualidade do sêmen criopreservado é indispensável para o sucesso de biotécnicas como a inseminação artificial. Sendo assim, é importante preservar ao máximo sua qualidade durante os processos de criopreservação e descongelamento (Holt, 2000).
Um dos problemas encontrados na criopreservação de sêmen é o estresse oxidativo que ocorre nos processos de refrigeração, congelamento e descongelamento, resultando em uma diminuição de qualidade quando comparada ao sêmen fresco (Maia e Bicudo, 2009). Em condições normais, a célula espermática produz e degrada espécies reativas de oxigênio (EROs), as quais são necessárias para a funcionalidade da

\footnotetext{
${ }^{*}$ Recebido em 9 de setembro de 2014 e aceito em 2 de julho de 2015. ***Dept. Clinica Médica Veterinária (CLIMEV), FAMEVZ, UFMT. Cuiabá-MT, Brasil.

****Dept. Ciências Básicas e Produção Animal (DCBPA), FAMEVZ, UFMT. Cuiabá-MT, Brasil.

****Departamento de Reprodução Animal, FMVZ-USP. São Paulo-SP, Brasil.

******Universidade Federal de São Paulo, UNIFESP. São Paulo-SP, Brasil.

******Mestrando no Programa de Pós Graduação em Ciência Animal, FAMEVZ, UFMT. Cuiabá-MT, Brasil.

*******Instituto Federal de Educação Ciência e Tecnologia, Campus São Vicente. Cuiabá-MT, Brasil.
}

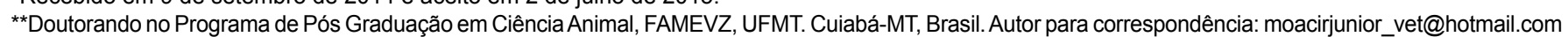


célula. É conhecida a participação do ânion superóxido e do peróxido de hidrogênio em mecanismos que culminam com a fertilização em diversas espécies. O problema é que a criopreservação normalmente acarreta um desbalanço entre a produção de EROs e sua eliminação pelo sistema antioxidante intra e extra celular, levando o sistema ao estado de estresse oxidativo (Maia e Bicudo, 2009).

Diversos estudos têm sido desenvolvidos buscando regular o controle hormonal do ciclo estral para o emprego da inseminação artificial em tempo fixo (IATF), no entanto, vale ressaltar que a qualidade do sêmen a ser utilizado na IATF é fundamental para se obter boas taxas de prenhez (Arruda et al., 2006).

Neste sentido, pesquisas têm sido realizadas com adição de tocoferol em diferentes concentrações ao meio diluidor (Beconiet al., 1993; Nasiri et al., 2012) ou suplementação oral (Hatamoto et al., 2006), buscando sua interação com radicais oxidantes, interrompendo a reação em cadeia da peroxidação lipídica da membrana, protegendo a célula do estresse oxidativo, e contra danos de membrana plasmática e acrossomal em diferentes espécies (Almeida e Ball, 2005; Silva et al., 2009).

Este trabalho foi realizado com o objetivo de avaliar a fertilidade do sêmen bovino após a adição de tocoferol ao meio diluidor de criopreservação, na taxa de prenhez após IATF.

\section{Material e métodos}

A coleta de sêmen foi realizada na Fazenda Paraíso Toca do Tatu, município de Santo Antônio do Leverger-MT. A coleta e congelamento do sêmen foram realizados a campo em fevereiro/2012 e a análise das amostras posteriormente nas dependências do dependências do Laboratório de Biotecnologia e Reprodução Animal da Universidade Federal de Mato Grosso - UFMT e no Laboratório de Andrologia da Universidade de São Paulo - USP.

Foram utilizados três reprodutores da raça Nelore (Bos taurus indicus) com idade média de 48 meses e peso vivo médio de $750 \mathrm{~kg}$, que se encontravam em repouso sexual, selecionados de acordo com a qualidade espermática (CBRA, 2013) de um lote de 9 touros adultos. Foi coletado um único ejaculado de cada reprodutor, distribuído aleatoriamente entre as fêmeas inseminadas artificialmente. Os animais foram mantidos em pasto de Brachiariabrizantha cv. Marandu, com mistura mineral comercial e água ad libitum, apresentavam boa condição corporal (escore 3,5 na escala de 1-5). A coletado ejaculado foi realizada através do método de eletroestimulação, em seguida alíquotas foram retiradas para a realização dos exames imediatos no sêmen. Ejaculados com valores inferiores a $60 \%$ de motilidade e com porcentagem de patologia espermática total superiores a $30 \%$ foram descartados (CBRA, 2013). Os ejaculados que estavam dentro dos parâmetros foram encaminhados para o congelamento manual, que foi realizado em laboratório montado em um local próximo à coleta, à temperatura ambiente.

No sêmen fresco foram avaliadas a motilidade, vigor e viabilidade espermática, integridade acrossomal, morfologia e concentração espermática. A motilidade e vigor espermáticos foram determinadas pela deposição de uma gota de sêmen entre lâmina e lamínula, sob microscopia em magnificação de $400 x$ com base na estimativa visual (CBRA, 2013). A viabilidade espermática foi avaliada utilizando a coloração eosina-nigrosina
(Who, 1992) e a integridade acrossomal através da coloração simples Fast-Green/Rosa-Bengala (Popeet al., 1991). A concentração espermática foi determinada pela contagem das células em câmara de Neubauer, após diluição (1:150) de uma alíquota de sêmen em formol salino. A morfologia espermática foi avaliada em preparações úmidas, entre lâmina e lamínula, em magnificação de 400x em microscopia de contraste de fase e classificados em defeitos menores, maiores e totais (CBRA, 2013).

Após avaliação da concentração da amostra seminal, cada ejaculado foi dividido em duas frações e diluído em extensor TRIS-citrato-gema $(3,187 \mathrm{~g}$ TRIS-hidroximetil amino metano; $1,78 \mathrm{~g}$ ácido cítrico mono-hidratado; $1,316 \mathrm{~g}$ frutose; $80 \mathrm{~mL}$ água destilada; $20 \mathrm{~mL}$ gema de ovo; $1000 \mu \mathrm{g} / \mathrm{mL}$ diluidor de estreptomicina; $1000 \mathrm{UI} / \mathrm{mL}$ diluidor de penicilina para cada $100 \mathrm{~mL}$ de diluidor) Fração $\mathrm{A}$ (sem glicerol). Logo após foram resfriadas em geladeira por 4 horas a uma temperatura de $4^{\circ} \mathrm{C}$. Em seguida foi adicionado o extensor TRIS-citrato-gema Fração B (8\% glicerol) na proporção 1:1.

$\mathrm{O}$ aditivo foi adicionado somente na diluição final, os dois tratamentos foram: Controle (sem aditivos) e Tocoferol (10 $\mathrm{mmol} /$ $\mathrm{mL}$ ). Após a adição do tocoferol, o sêmen foi envasado em palhetas de $0,5 \mathrm{~mL}$, sendo a concentração final fixada em 25 milhões de espermatozoides viáveis por palheta. Em seguida foram colocadas em estantes a $5 \mathrm{~cm}$ acima do nitrogênio líquido e mantidas no vapor de nitrogênio por 15 minutos, logo após mergulhadas em nitrogênio líquido $\left(-196^{\circ} \mathrm{C}\right)$ e posteriormente armazenadas em botijões criogênicos. $\mathrm{O}$ descongelamento do sêmen para avaliações foi realizado em banho Maria a $37^{\circ} \mathrm{C}$ por 30 segundos.

Após o descongelamento das amostras foram retiradas alíquotas para avaliação da motilidade espermática e avaliação do estresse oxidativo. Para confecção das lâminas coradas foi utilizado $300 \mu \mathrm{L}$, lavado duas vezes com $1600 \mu \mathrm{L}$ de PBS, homogeneizando e centrifugando $(800 \times \mathrm{g}, 10 \mathrm{~min})$, com a finalidade de remover os resquícios de diluidor. Após esse processo os espermatozoides foram ressuspensos em $200 \mu \mathrm{L}$ de PBS e lâminas foram confeccionadas para avaliação da integridade da membrana plasmática (Who, 1992), integridade acrossomal (Popeet al., 1991) e atividade citoquímica mitocondrial (Hrudka, 1987).

Para avaliação da motilidade e vigor espermáticos foi utilizado microscopia, com base na estimativa visual (CBRA, 2013).

$A$ resistência do espermatozoide ao estresse oxidativo foi mensurada pela técnica de substâncias reativas ao ácido tiobarbitúrico(TBARS) induzido. Esse procedimento é conhecido como lipoperoxidação induzida, e tem como objetivo quantificar o potencial que a amostra teria de gerar os radicais em questão. Após o descongelamento uma alíquota de $200 \mu \mathrm{L}$ foi lavada duas vezes com a finalidade de remover os resquícios de diluidor, acrescendo $1600 \mu \mathrm{L}$ de PBS, homogeneizando, centrifugando $(800 \times \mathrm{g}, 10 \mathrm{~min})$ e retirando-se $1600 \mu \mathrm{L}$ do preparo. Após a lavagem foi acrescido sulfato de ferro $(50 \mu \mathrm{L}$, $4 \mathrm{mM})$ e ascorbato de sódio $(50 \mu \mathrm{L}, 20 \mathrm{mM})$ aos $200 \mu \mathrm{L}$ da suspensão espermática. Em seguida a amostra foi incubada em microtubos por uma hora e meia a $37^{\circ} \mathrm{C}$ (banho-maria) com a tampa aberta. Após esse período a amostra foi misturada a $600 \mu \mathrm{L}$ de ácido tricloroacético (TCA) 10\%, homogeneizada e centrifugada $\left(18,000 \times \mathrm{g}, 15 \mathrm{~min}, 15^{\circ} \mathrm{C}\right)$, para a precipitação de 
proteínas. Após centrifugação uma alíquota do sobrenadante foi recuperado $(800 \mu \mathrm{L})$ e congelado para posterior análise. As amostras congeladas foram descongeladas em estufa a $37^{\circ} \mathrm{C}$ por 10 minutos e misturadas com $800 \mu \mathrm{L}$ de ácido tiobarbitúrico (TBA) $1 \%$, em minitubos e incubadas em água fervente (90 a $100^{\circ} \mathrm{C}$ ) por $15 \mathrm{~min}$. A absorvância das amostras foram então quantificadas utilizando-se um espectrofotômetro (FEMTO 800 $\mathrm{XI}$ ) em comprimento de onda de $532 \mathrm{~nm}$. Os resultados foram comparados à absorvância de uma curva padrão previamente preparada com uma solução padrão de malondealdeído(MDA). A concentração de TBARS foi determinada utilizando um valor de $1,56 \times 10^{5} \mathrm{M} / \mathrm{mL}$ com o coeficiente de extinção molar do MDA (Buege eAust, 1978). O índice de peroxidação lipídica foi descrito em nanogramas de TBARS por $10^{6}$ espermatozoides.

Previamente ao início do protocolo de IATF, todos os animais foram classificados por escore de condição corporal, apresentando em média o escore $3(1-5)$ e submetidos a exame ginecológico, por ultrassonografia, sendo que nenhuma fêmea apresentou qualquer distúrbio ginecológico que comprometesse o experimento. Estas fêmeas eram provenientes do XXX, criadas em sistema extensivo, mantidas em pasto de Brachiaria humidícola cv. humidícola, com mistura mineral comercial e água ad libitum.

Para a sincronização do estro, cada animal recebeu no dia zero (D0) - Primeiro lote em 13/02/2012 e segundo lote 03/04/2012, um dispositivo intravaginal de liberação lenta de progesterona contendo $1 \mathrm{~g}\left(\mathrm{DIB}^{\circledR}\right)$, mantido por 9 dias. No momento da implantação do dispositivo (D0), os animais receberam $2,5 \mathrm{mg}$ de benzoato de estradiol - BE (Estrogin $\left.{ }^{\circledR}\right)$, por via intramuscular (IM) profunda.

No momento da retirada dos dispositivo (D9), todos os animais receberam, por via IM profunda, 25mg de dinoprost (Lutalyse ${ }^{\circledR}$ ), 500 UI de gonadotrofina coriônica eqüina $\left(\right.$ Novormon $^{\circledR}$ ) e $1 \mathrm{mg}$ de cipionato de estradiol (E.C.P. ${ }^{\circledR}$ ).

No D11, 75fêmeas nelore (primeiro lote) foram divididas aleatoriamente em dois Grupos: Grupo controle, ou seja, inseminadas com sêmen criopreservado com TRIS-citratogema sem o uso de aditivos $(n=41)$, sendo este constituído por 19 vacas paridas e 22 vacas solteiras. E o Grupo tocoferol, inseminadas com sêmen criopreservado com adição de tocoferol/10mmol $(n=34)$, sendo constituído por 10 vacas paridas e 24 vacas solteiras.

As fêmeas que não emprenharam no primeiro lote foram ressincronizadas utilizandose o mesmo protocolo, sendo o segundo lote constituído por 36 fêmeas divididas aleatoriamente em Grupo controle $(n=17)$, sendo este constituído por oito vacas paridas e 9 vacas solteiras. E o Grupo tocoferol $(n=19)$, sendo constituído por oito vacas paridas e 11 vacas solteiras.

Um total de 111 fêmeas com idades variando entre 48 e 96 meses foram inseminadas, das quais 45 eram vacas lactantes entre 30 e 120 dias pós parto e 66 vacas solteiras. Destas 25 fêmeas foram inseminadas do touro 01 (12-tocoferol e 13-controle), 33 fêmeas do touro 02 (15-tocoferol e 18-controle) e 53 fêmeas do touro 03 (26-tocoferol e 27-controle).

Todas as fêmeas foram examinadas por ultrassonografia transretal para diagnóstico de gestação 35 dias após cada protocolo de IATF, utilizando um aparelho Aloka SSD-500, equipado com sonda de arranjo linear de $5 \mathrm{MHz}$. A detecção de uma vesícula embrionária com embrião viável (presença de batimento cardíaco) foi considerada como indicativo de prenhez, sendo a taxa de prenhez obtida dividindo-se o número de fêmeas gestantes pelo número total de fêmeas inseminadas artificialmente.

O experimento foi realizado em delineamento inteiramente casualizado, e a taxa de prenhez comparada pelo teste quiquadrado. Os dados foram analisados com nível de significância de $5 \%$, sendo utilizado o programa estatístico SAS (SAS, 2001).

\section{Resultados}

Os parâmetros seminais de sêmen fresco e pós-descongelamento dos três touros utilizados na IATF estão demonstrados na Tabela 1.

Tabela 1: Valores obtidos dos parâmetros de motilidade e vigor com base na estimativa visual, concentração espermática e morfologia espermática no sêmen fresco e motilidade e vigor com base na estimativa visual, integridade do acrossomo e da membrana plasmática espermática, da atividade citoquímica mitocondrial e nível de peroxidação lipídica após descongelamento de amostras do sêmen bovino utilizado na IATF

\begin{tabular}{lccc}
\hline & \multicolumn{3}{c}{ Sêmen fresco } \\
\cline { 2 - 4 } \multicolumn{1}{c}{ Parâmetros } & Touro 1 & Touro 2 & Touro 3 \\
\hline MOT \% & 80 & 85 & 80 \\
Vigor $(1-5)$ & 3 & 3 & 3 \\
{$\left[\right.$ ] sptz/ml $\times 10^{6}$} & 380 & 400 & 600 \\
DEFTOT & 12 & 9 & 19 \\
\hline
\end{tabular}

\begin{tabular}{lcccccc}
\hline & \multicolumn{7}{c}{ Após descongelamento } \\
& \multicolumn{2}{c}{ Touro 1 } & \multicolumn{2}{c}{ Touro 2 } & \multicolumn{2}{c}{ Touro 3 } \\
\cline { 2 - 7 } & Controle & Tocoferol & Controle & Tocoferol & Controle & Tocoferol \\
\hline EOS \% & 60 & 73 & 31 & 64 & 25 & 28 \\
POPE \% & 74 & 87 & 82 & 91 & 60 & 67 \\
DAB I & - & - & 72 & 38 & 43 & 30 \\
DAB II & - & - & 22 & 46 & 14 & 20 \\
DAB III & - & - & 4 & 12 & 9 & 13 \\
DAB IV & - & - & 2 & 4 & 34 & 37 \\
EOX & - & - & 80,546 & 116,781 & 83,244 & 86,713 \\
MOT \% & 50 & 60 & 45 & 55 & 30 & 30 \\
Vigor (1-5) & 3 & 3 & 3 & 3 & 3 & 3 \\
\hline
\end{tabular}

DEFTOT: Defeitos espermáticos totais; EOS: Integridade da membrana plasmática; POPE: integridade do acrossomo; Classes de espermatozoides: (DAB I) todas mitocôndrias ativas, (DAB II) predominância de mitocôndrias ativas, (DAB III) menos da metade das mitocôndrias ativas, (DAB IV) todas mitocôndrias inativas; EOX: concentração de TBARS (ng/106 sptz) induzido; MOT: motilidade com base na estimativa visual. 
A adição de $10 \mathrm{mmol} / \mathrm{mL}$ de tocoferol no meio extensor do sêmen criopreservado não influenciou $(P>0,05)$ na taxa de prenhez após IATF em comparação ao grupo controle (sem aditivos) para as médias do primeiro lote sincronizado $(n=75)$, segundo lote sincronizado $(n=36)$ e para a média geral dos lotes $(n=111)$ (Figura 1).

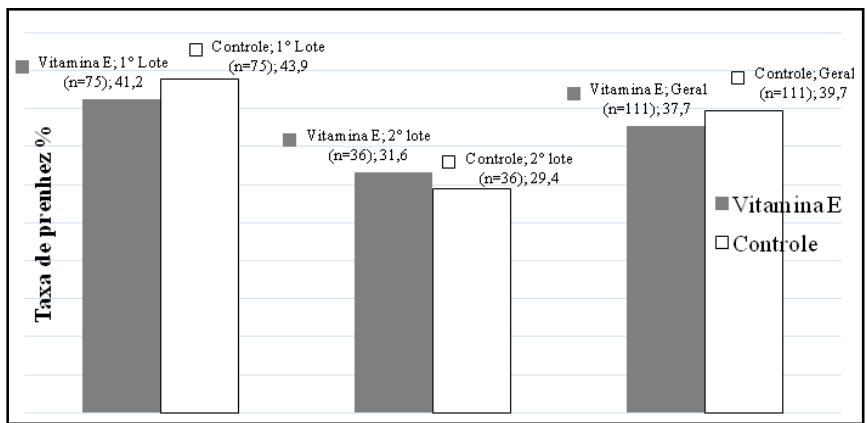

Figura 1:Taxa de prenhez em fêmeas Nelore após IATF em função do efeito do tratamento com antioxidante $(10 \mathrm{mmol} / \mathrm{mL}$ tocoferol) em comparação ao grupo controle (sem aditivos) para os dois lotes sincronizados e média geral do experimento

Quando comparadas entre categorias animal: vacas $(n=66)$ e vacas paridas $(\mathrm{n}=45)$, a adição de $10 \mathrm{mmol} / \mathrm{mL}$ de tocoferol no meio extensor do sêmen criopreservado não influenciou $(P>0,05)$ na taxa de prenhez após IATF em comparação ao grupo controle (sem aditivos) (Figura 2).

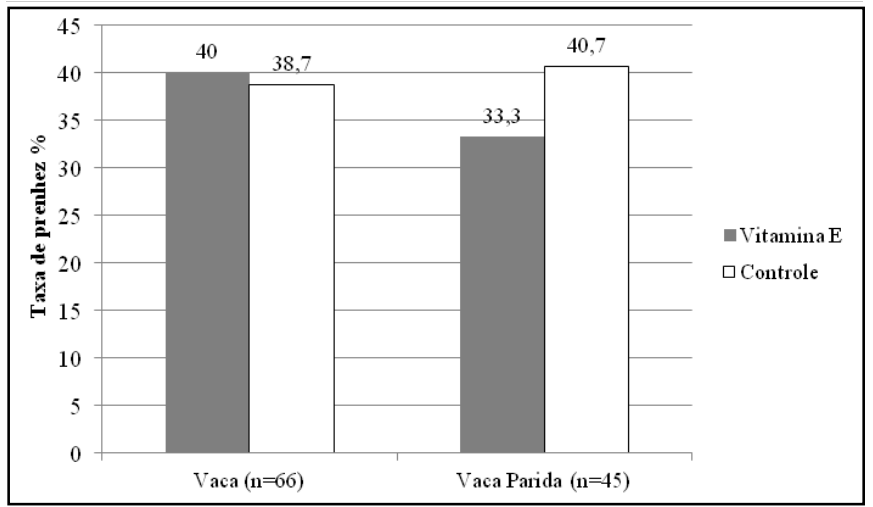

Figura 2: Taxa de prenhez em fêmes Nelore após IATF em função do efeito do tratamento com antioxidante $(10 \mathrm{mmol} / \mathrm{mL}$ tocoferol) em comparação ao grupo controle (sem aditivos) para as diferentes categorias animal: vacas e vacas paridas

A adição de $10 \mathrm{mmol} / \mathrm{mL}$ de tocoferol no meio extensor do sêmen criopreservado não influenciou $(P>0,05)$ na taxa de prenhez após a IATF entre os três touros avaliados, e entre os tratamentos para cada animal (Tabela 2).

\section{Discussão}

Foi verificado que a adição de tocoferol ao diluidor de criopreservação do sêmen não proporcionou incremento na taxa de prenhez após a IATF em comparação ao grupo controle, quando se comparou as médias dos lotes separados ou a média geral (Figura 1), corroborando com os resultados encontrados por Borges (2008) que não verificou diferença na taxa de prenhez utilizando
0,2 $\mathrm{mmol} / \mathrm{mL}$ de Trolox (antioxidante semelhante ao tocoferol, porém solúvel em água) ao meio extensor de sêmen utilizado na inseminação artificial em bovinos em comparação ao grupo controle ( $58 \%$ vs $58,7 \%$ ), porém com inseminação artificial após observação de cio. Também não foi verificado efeito do tratamento quando os animais foram comparados de acordo com sua categoria animal (Figura 2). Apesar de ser considerado um nutriente indispensável à reprodução, os mecanismos e funções fisiológicas no qual o tocoferol desempenha, ainda não são completamente evidenciados (Brigelius-Flohé e Traber, 1999). Estudos têm sido realizados buscando demonstrar o efeito da adição do tocoferol seja por suplementação oral dos animais (Marinho, 2009) ou sua adição ao diluidor do sêmen para refrigeração (Almeida e Ball, 2005) e criopreservação (Soares, 2012) para melhorar a qualidade espermática em diversas espécies.

Frequentemente são utilizados testes in vitro na determinação da qualidade do sêmen para a sua utilização em inseminação artificial ou em procedimentos de biotecnologia de embriões, devido à correlação existente entre qualidade do sêmen analisado in vitro e sua fertilidade in vivo em bovinos (Perumalet al., 2011) e ovinos (Bicudo et al., 2007), mas, apesar de apresentarem boa estimativa da capacidade fecundante, estes testes não são tão eficientes em predizer a capacidade fertilizante do espermatozoide como os testes in vivo (ex. inseminação artificial) (Borges, 2008).

Faltam informações na literatura a respeito da utilização de antioxidantes não enzimáticos em testes in vivo em bovinos, principalmente na IATF, era esperado que a adição de tocoferol ao meio crioprotetor do sêmen provocasse um efeito benéfico, representado por um aumento na taxa de prenhez após IATF, uma vez que o tocoferol age protegendo a membrana plasmática, diminuindo a suceptibilidade a lipoperoxidação e preservando a viabilidade dos espermatozoides durante a criopreservação (Beconiet al., 1993), características essas, fundamentais ao sêmen utilizado em programas de IATF, visto que os protocolos disponíveis para sincronização de cio podem apresentar uma dispersão quanto ao momento da ovulação, afetando a taxa de prenhez.

No entanto, a eficiência de um tratamento com antioxidantes depende ainda de fatores como a presença de agentes oxidativos suficientes para causar um desbalanço do sistema antioxidante, sendo assim, amostras que não estejam sobre os

Tabela 2: Taxa de prenhez em fêmeas Nelore após IATF em função do efeito do tratamento com antioxidante $(10 \mathrm{mmol} / \mathrm{mL}$ tocoferol) em comparação ao grupo controle (sem aditivos) para os três Touros utilizados e média geral para cada Touro

\begin{tabular}{cccc}
\hline Touro & Grupos & Taxa prenhez $(\%)$ & $\begin{array}{c}\text { Número de animais } \\
\text { inseminados }\end{array}$ \\
\hline \multirow{2}{*}{01} & Tocoferol & 41,7 & 12 \\
& Controle & 38,5 & 13 \\
& Total & 40,0 & 25 \\
02 & Tocoferol & 46,7 & 15 \\
& Controle & 44,4 & 18 \\
& Total & 45,5 & 33 \\
03 & Tocoferol & 30,8 & 26 \\
& Controle & 37,0 & 27 \\
& Total & 34,0 & 53 \\
\hline
\end{tabular}

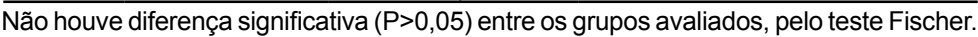


efeitos de estresse oxidativo, podem não apresentar o efeito desejado após o tratamento.

Segundo o NRC (1996), a exigência diária de tocoferol para bovinos de corte adultos varia entre 50 a $100 \mathrm{UI} / \mathrm{dia}$, e na maioria das gramíneas não são encontrados valores significativos deste composto, sendo necessária uma suplementação adicional, uma vez que ruminantes não são capazes de sintetizá-las (Balletet al., 2000). No entanto, é provável que nas condições experimentais, os touros utilizados para coleta de sêmen, estivessem suprindo suas exigências diárias de tocoferol através da pastagem e suplementação ingerida.

\section{Referências}

ALMEIDA, J.; BALL, B.A. Effect of a-tocopherol and tocopherol succinate on lipid peroxidation in equine spermatozoa.Animal reproduction Science.v.87, p.321-337, 2005.

ARRUDA, R.P.; CELEGHINI, E.C.C.; ANDRADE, A.F.C.; RAPHAEL, C.F.; PERES, K.R.; NEVES, L.C. Influência da qualidade do sêmen nos resultados de prenhez em programas de IATF e TETF. Biotecnologia da reprodução em bovinos. In: Anais do $2^{\circ}$ Simpósio Internacional de Reprodução Animal Aplicada. São Paulo. p.157-164, 2006.

BALLET, N. J.; et al. Vitamins in forages. In: GIVENS, D.I.; et al. Forage Evaluation in Ruminant Nutrition. CAB International, Wallingford, UK, 2000, $373 \mathrm{p}$.

BECONI, M.T.; FRANCIA, C.R.; MORA, N.G.; AFFRANCHIO, M.A. Effect of natural antioxidants on frozen bovine semen preservation. Theriogenology. v. 40, p. 841-851, 1993.

BICUDO, S.D.; AZEVEDO, H.C.; MAIA, S.M.; GREEN, R.E.; RODELLO, L.; MEIRA, C. Avanços na criopreservação do sêmen ovino visando sua aplicação em programas de inseminação artificial e em biotecnologias com embriões. Acta Scientiae Veterinariae. v.35, n.3, p.787-798, 2007.

BORGES, J. C. Efeito da utilização de antioxidante no diluidor para a criopreservação de sêmen bovino avaliado através de testes complementares, inseminação artificial e fecundação in vitro. 2008. 70 f. Tese (Doutorado) - Faculdade de Ciências Agrárias e Veterinárias, Universidade Estadual Paulista, Jaboticabal, 2008.

BRIGELIUS-FLOHÉ, R.; TRABER, M.G. Vitamin E: function and metabolism. The FASEB Journal. v. 13, p. 1145-1155 , 1999.

BUEGE, J. A.; AUST, S. D. Microsomal lipid peroxidation.Methods of Enzymology, v. 52, n. 1, p. 302-310, 1978.

CBRA. Colégio Brasileiro de Reprodução Animal. Manual para exame andrológico e avaliação de sêmen animal. 3. ed. Belo Horizonte, 2013, $104 \mathrm{p}$.

HATAMOTO, L. K.; BAPTISTA SOBRINHO, C. A.; NICHI, M.; BARNABE, V. H.; BARNABE, R. C.; CORTADA, C. N. M. Effects of dexamethasone treatament (to mimic stress) and Vitamin $\mathrm{E}$ in oral supplementation on the spermiograma and on plasma spontaneous lipid peroxidation and antioxidant enzyme activies in dogs. Theriogenology v. 66, p. 1610-1614, 2006.

HOLT, W.V. Basic aspects of frozen storage of semen.Animal Reproduction Science.v.62, p. 3-22, 2000.

HRUDKA, F. Cytochemical and ultracytochemical demonstration of cytochrome-c oxidase in spermatozoa and dynamics of changes accompanying ageing or induced by stress. InternationalJournalofAndrology, v. 10, n. 6, p. 809-828, 1987.
E ainda, uma vez que não foi verificada diferença entre os touros utilizados para os parâmetros seminais avaliados, a utilização do tocoferol ao meio diluidor não trouxe os benefícios esperados, que representaria maior taxa de prenhez quando comparado ao grupo controle.

\section{Conclusões}

A adição de $10 \mathrm{mmol}$ de tocoferol ao meio crioprotetor do sêmen não melhorou a qualidade do sêmen congelado, nem a taxa de prenhez após a inseminação artificial em tempo fixo na espécie bovina.

MAIA, M.S.; BICUDO, S.D.; Radicais livres, antioxidantes e função espermática em mamíferos: uma revisão. Revista Brasileira Reprodução Animal. Belo Horizonte, v. 33, n. 4, p.183193, 2009.

MARINHO, W.A.S. Suplementação oral com vitamina $E$ para touros Brangus: Integridade da membrana espermática, qualidade seminal a fresco e pós-congelamento, 2009. 70 f. Dissertação (Mestrado em Ciência Animal), Faculdade de Agronomia e Medicina Veterinária, Universidade Federal de Mato Grosso, Cuiabá, 2009.

NASIRI, A.H.; TOWHIDI, A.; ZEINOALDINI, S. Combined effect of DHA and a-tocopherol supplementation during bull semen cryopreservation on sperm characteristics and fatty acid composition. Andrologia. v. 44, p. 550-555, 2012.

NATIONAL RESEARCH COUNCIL.Nutrient Requirements of Beef Cattle.Washington, D.C. National Academy of Sciences, 7. ed., 242 p., 1996.

PERUMAL, P.; SELVARAJU, S.; SELVAKUMAR, S.; BARIK, A.K.; MOHANTY, D.N.; DAS, S.; MISHRA, P.C. Effect or prefreeze addition of cysteine hydrochloride and reduced glutathione in semen of crossbred jersey bulls on sperm parameters and conception rates. Reproduction in Domestic Animals.v. 46, p. 636641, 2011.

POPE, C. E.; ZHANG, Y. Z.; DRESSER, B. L.A simple staining method for evaluating acrossomal status of cat spermatozoa. Journal of Zoo and Wildlife Medicine, v. 22, n. 1, p. 87-95, 1991.

SAS (2001; SAS. The statistical analyse systems for windows: version 8. Cary, 1999-2001. CD-Rom).

SILVA, K.M.G.; MORAES, T.A.P.; SILVA, E.C.B.; GAMBOA, S.C.; GUERRA, M.M.P. Efeito da adição de trolox e pentoxifilina na motilidade, integridade do acrossoma e do DNA de espermatozoides equinos após descongelação. Arquivo Brasileiro de Medicina veterinária e Zootecnia. v. 61, n.1, p. 42-49, 2009.

SOARES, L.C.M. Vitamina $E$ e qualidade do sêmen bovino fresco e criopreservado. 2012. 66 f. Dissertação (Mestrado em Ciência Animal), Faculdade de Agronomia e Medicina Veterinária, Universidade Federal de Mato Grosso, Cuiabá, 2012.

WHO.World Health Organization.WHO laboratory manual for the examination of human semen and semen - cervical mucus interaction. Cambridge: The Press Syndicate of the University of Cambridge, 1992. p. 120. 